\title{
Regorafenib-to-trifluridine/tipiracil Versus the Reverse Sequence for Refractory Metastatic Colorectal Cancer Patients: A Multicenter Retrospective Real-life Experience
}

\author{
CARLO SIGNORELLI ${ }^{1}$, DONATELLO GEMMA ${ }^{2}$, ROBERTA GRANDE ${ }^{3}$, SALVATORE DE MARCO $^{4}$, \\ ROSA SALTARELLI ${ }^{5}$, MARIA GRAZIA MORANDI ${ }^{6}$, GIAN PAOLO SPINELLI ${ }^{7}$, FEDERICA ZORATTO ${ }^{8}$, \\ ISABELLA SPERDUTI $^{9}$, MARIO GIOVANNI CHILELLI ${ }^{1}$, ANNA CERIBELLI $^{6}$ and ENZO MARIA RUGGERI ${ }^{1}$ \\ ${ }^{1}$ Medical Oncology Unit, Belcolle Hospital, ASL Viterbo, Viterbo, Italy; \\ ${ }^{2}$ Medical Oncology Unit, ASL Frosinone, Sora, Italy.; \\ ${ }^{3}$ Medical Oncology Unit, ASL Frosinone, Frosinone, Italy; \\ ${ }^{4}$ Medical Oncology Unit, San Camillo-Forlanini Hospital, Rome, Italy; \\ ${ }^{5}$ UOC Oncology, San Giovanni Evangelista Hospital, ASL RM5, Rome, Italy; \\ ${ }^{6}$ Medical Oncology Unit, San Camillo de Lellis Hospital, ASL Rieti, Rieti, Italy; \\ ${ }^{7}$ UOC Oncology, University of Rome "La Sapienza", ASL Latina, Aprilia, Italy; \\ ${ }^{8}$ Medical Oncology Unit, ASL Latina, Latina, Italy; \\ ${ }^{9}$ Biostatistics Unit, IRCCS Regina Elena National Cancer Institute, Rome, Italy
}

\begin{abstract}
Background/Aim: Regorafenib (REG) and trifluridinel tipiracil (FTD/TPI) have have been shown to improve overall survival in patients with refractory metastatic colorectal cancer. The aim of our study was to evaluate the efficacy and safety profiles of these agents administered in sequence in real world practice. Patients and Methods: Clinical data of patients treated beyond the $2 \%$ line with REG or FTD/TPI between January 2016 and August 2020, were retrospectively collected from eight institutes in the Lazio Region. Results: We included 49 patients treated with both drug sequences. A total of 28 G3/G4 toxicity events (53.8\%) were recorded in the FTD/TPI-to-REG sequence vs. 24 $(46.1 \%)$ in the reverse sequence. Median overall survival for the patients included in the FTP/TPI-to-REG group was 20 months (95\%CI=16.7-23.3) vs. 27 months in the reverse group (95\%CI=17.8-36.2). The disease control rate was $45.0 \%$ for patients treated with the REG-to-FTD/TPI sequence vs. $24.1 \%$ in those treated with the FTD/TPI-to$R E G$ sequence ( $p=0.18)$. Conclusion: The sequence REG-to-
\end{abstract}

This article is freely accessible online.

Correspondence to: Dr. Carlo Signorelli, Medical Oncology Unit, Belcolle Hospital, ASL Viterbo, Strada Sammartinese snc, 01100 Viterbo, Italy. Tel/Fax: +39 0766846291, e-mail: carlo.signorelli@asl.vt.it

Key Words: Regorafenib, trifluridine/tipiracil, refractory metastatic colorectal cancer, real-world practice.
FTD/TPI and vice versa can extend survival, whereas only REG-to-FTD/TPI stabilizes cancer growth.

Colorectal cancer (CRC) is the third most common cancer and the second leading cause of cancer-associated death. In 2020, worldwide, 600,896 new cases in men $(10.6 \%$ of all incident cancer cases in men) and 547,619 new cases in women $(9.4 \%$ of all incident cancer cases in women) were recorded (1). At diagnosis, approximately $20 \%$ of patients (pts) with CRC present with synchronous metastatic disease and between $50 \%$ and $60 \%$ of patients develop metastases over their treatment course. As a result of remarkable improvements in the development of combination chemotherapy regimens comprising cytotoxic agents (e.g., fluoropyrimidine, oxaliplatin, and irinotecan) and molecular targeted therapies (e.g., bevacizumab, ramucirumab, ziv-aflibercept, cetuximab, panitumumab, and regorafenib) for the treatment of patients with metastatic colorectal cancer (mCRC) in the past two decades, substantial improvements have been observed, with the median overall survival (OS) from firstline chemotherapy reaching approximately 30 months in randomised clinical trials. Based on the knowledge of the immunogenic microenvironment of tumours with high microsatellite instability (MSI-H), recent studies showed that the mismatch repair (MMR) status is predictive of the clinical benefit of pembrolizumab $(2,3)$. Despite these advances in systemic therapy, mCRC has still poor prognosis, with 5-year OS rates of less than $20 \%$. Furthermore, it must be noted that approximately $30 \%$ of patients already undergoing chemotherapy beyond the standard second-line regimens 
continue to have a good performance status and can be good candidates for further treatments (4-11). In late-lines, therapeutic options recommended by the European Society of Medical Oncology (ESMO) and the National Comprehensive Cancer Network (NCCN) guidelines include agents such as REG and FTD/TPI $(12,13)$. Patients with refractory mCRC rarely undergo third-line or subsequent treatment. This strategy could have a negative impact on their OS. To date, FTD/TPI (a combination of trifluridine and tipiracil hydrochloride; trifluridine is a thymidine-based nucleic acid analog, and tipiracil hydrochloride is a thymidine phosphorylase inhibitor which enables the maintenance of high trifluridine concentration) and REG (a multikinase inhibitor that is active against several angiogenic receptor tyrosine kinases, oncogenic receptor tyrosine kinases RTKs, stromal receptor tyrosine kinases, and intracellular signaling kinases) are the two key new treatment options with statistically significant improvements in OS, progression-free survival (PFS) and disease control in randomized phase III trials $(10,11,14,15)$. The tolerance profiles are different. However, these two drugs are considered clinically insignificant based on the modest benefits in OS and PFS. A recent multicenter observational study highlighted the clinical benefit and tolerability of these drugs in real clinical practice and the criteria for their choice (16). However, some questions remain to be answered including which subpopulations of pts might benefit most from treatment with these drugs compared to the best supportive care, which is the most appropriate mode of administration for pts with advanced disease, and which subpopulations of pts might benefit from vital treatment with these drugs, compared to the best supportive therapy alone (1722). REG and FTD/TPI have not been directly compared in head-to-head trials (23-29). So it is unclear and under discussion which drug should be administered first. Little insights are available to guide the selection and sequence of treatments for this patient population. The aim of our multi-institutional study was to retrospectively evaluate, in real-world practice, the impact on clinical outcomes and safety of FTD/TPI and REG administered sequentially versus in the reverse sequence in patients with mCRC refractory to standard chemotherapies.

\section{Patients and Methods}

For this analysis, clinical data of 49 pts with mCRC who were treated with REG-to-FTD/TPI or FTD/TPI-to-REG sequences beyond the $2^{\text {nd }}$ line at eight Lazio region centers from January 2016 and August 2020, were retrospectively identified and collected from the electronic database pertaining to patient and disease characteristics, treatment and outcomes. All data were anonymized and pts were defined by a number and their initials to ensure the protection of sensitive data. The data manager was the principal investigator. The principal investigator, his collaborators and the biostatistician had access to the entire database in compliance with the provisions of the law. The study design is illustrated in Figure 1. Patients were categorized into two groups based on the sequence of treatment they received: FTD/TPI-to-REG and REG-to-FTD/TPI groups. The enrolled pts met the following inclusion criteria: 1) histologically confirmed stage IV adenocarcinoma of the colon or rectum with unresectable metastatic disease at diagnosis or in the postoperative follow-up; 2) pts with synchronous or metachronous metastases; 3 ) previous administration of at least two prior regimens of standard chemotherapies with fluoropyrimidine, irinotecan, oxaliplatin, and anti-VEGF antibody (bevacizumab and aflibercept), or anti-epidermal growth factor receptor (EGFR) antibody (cetuximab or panitumumab); 4) pts with metastatic colorectal adenocarcinoma treated with late-lines therapy with REG followed by FTD/TPI or vice versa; 5) Eastern Cooperative Oncology Group performance status (ECOG PS) of 0 to 2; 7) known RAS mutational status; 8) adequate organ function at the start of the treatment. Patients previously treated with REG or FTD/TPI or with nonmetastatic colorectal cancer, or with metastatic colorectal cancer under treatment with first or second line regimens, or with primary colon tumors treated with curative intent or with history of uncontrolled concomitant pathologies were excluded. The endpoints investigated were: OS defined as the time calculated from the initiation of treatment with the therapeutic sequence REG-toFTD/TPI or the reverse sequence FTD/TPI-to-REG, until death from any cause; PFS as the time from initiation of treatment with the REG-to-FTD/TPI therapeutic sequence or the reverse FTD/TPIto-REG sequence, until disease progression or death; objective response rate (ORR) that includes the complete and partial responses obtained with each therapeutic sequence was evaluated according to the RECIST criteria and disease control rate (DCR) as the sum of complete, partial and stable disease rates. Informed consent was waived due to the nature of the retrospective study and confidentiality was maintained for patient data. Adverse events were assessed using the National Cancer Institute Common Terminology Criteria for Adverse Events, version 4.0. Patients were followed until the $31^{\text {st }}$ of August 2020. All procedures for this retrospective observational study, which was conducted in accordance with the Declaration of Helsinki, were approved by the Lazio 1 Ethics Committee (approval no. 239/CE Lazio 1). Descriptive statistics were used to summarize relevant information about the study. Chisquare and Fisher Exact tests were used to evaluate possible associations. PFS and overall survival (OS) were calculated by the Kaplan-Meier product-limit method. Log-rank test was used to assess differences between subgroups. Significance was defined at p below the 0.05 level. The SPSS (version 21.0) statistical program was used for all the statiscal analyses. FTD/TPI was administered at $35 \mathrm{mg} / \mathrm{m}^{2}$ orally, twice daily, on days $1-5$ and $8-12$ with 2 days of rest, for 2 weeks, followed by a 14-day rest period in each month. REG was administered at $160 \mathrm{mg}$ as a standard dose, once daily for 21 days of a 28-day cycle; the dose-escalation strategy of regorafenib by physician decision (starting dose $80 \mathrm{mg} /$ day orally with weekly escalation, per $40 \mathrm{mg}$ increment, to $160 \mathrm{mg} /$ day) was permitted if no significant drug-related adverse events occurred (16). Treatments were stopped due to tumor progression, severe toxicity, or due to clinical decision for any reason.

\section{Results}

The baseline patient's characteristics and treatment data regarding all eligible pts are summarized in Table I. After applying all inclusion and exclusion criteria, 49 pts were included: 29 pts $(59.1 \%)$ treated with FTD/TPI-to-REG sequence and 20 pts (40.8\%) with the REG-to-FTD/TPI 


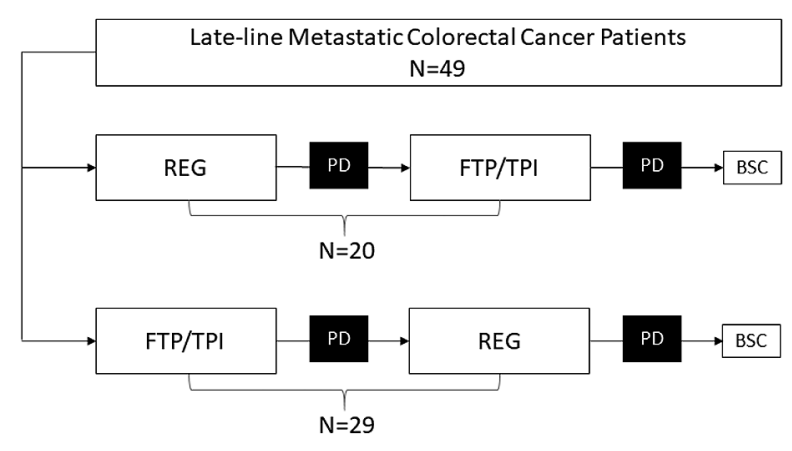

Figure 1. Study design.

reverse sequence. 32 pts $(65.3 \%)$ were males and $17(34.6 \%)$ were female, with a median age of 66 years (ys) (range $=45$ $83 \mathrm{ys})$; median duration of follow up was 10 mos (4-54); median number of cycles was 3 (1-15). An average of 8.3 cycles per patient were administered. At baseline, ECOG PS ranged between $0(40.8 \%)$ and $2(8.1 \%) .61 .2 \%$ of pts had a mutant global RAS tumour status. $34.6 \%$ of pts aged $>70 \mathrm{yrs}$ and $(41.1 \%$ in the REG-to-FTD/TPI group and $58.8 \%$ in the reverse group) and $65.3 \%$ aged $\leq 70$ yrs $(40.6 \%$ treated with the REG-to-FTD/TPI sequence and $59.3 \%$ with the reverse sequence). In the FTD/TPI-to-REG group, 12 pts had a PS 0 , 14 pts had a PS 1 and 3 pts had a PS 2, while in the reverse sequence group, 8 pts had PS 0,11 pts had a PS 1 , and only 1 patient had a PS 2 . Most pts $(63.2 \%)$ had received at least 2 active lines of treatment while $36.7 \%$ of pts had received 3 lines of therapy prior to the above sequences. Male gender was by far prevalent in the FTD/TPI-to-REG sequence group ( $79.3 \%$ vs. $20.6 \%$ ), whereas in the reverse sequence, female was slightly higher with $55 \%$ vs. $45 \%$. Regarding the site of the primary tumor, in the REG-to-FTD/TPI group the left side prevailed $(55.0 \%)$ unlike the right side which predominated with $55.1 \%$ in the reverse therapeutic sequence. Metastatic sites with hepatic involvement were more numerous in pts treated with the REG-to-FTD/TPI sequence (45.0\%) unlike in the FTD/TPI-to-REG group where pts had more nonhepatic secondaryarities (58.6\%). Bevacizumab was the most administered biological agent in $1^{\text {st }}(55 \%$ and $75 \%)$ and 2 nd line $(37.9 \%$ and $40.0 \%)$ preceding both therapeutic sequences. Median REG-to-FTD/TPI and FTD/TPI-to-REG treatment duration was 9 mos for both with a range of 5-36 mos and 4-15 mos, respectively. Regardless of the sequence adopted, to increase tolerability without decreasing efficacy, a proportion of pts received REG with a reduced-dose from the beginning: $63.2 \%$ of pts started with the initial dose of $80 \mathrm{mg}$ (mPFS $8 \mathrm{mos}$ ), $14.2 \%$ with the $120 \mathrm{mg}$ initial dose (mPFS $12.5 \mathrm{mos}$ ), whereas $22.4 \%$ of pts started with the full dose of $160 \mathrm{mg}$ (mPFS 9 mos). Table II shows the data on drug-related adverse events. Regarding noteworthy G3/G4 toxicities, 28 events $(53.8 \%)$ were recorded in FTD/TPI-toREG vs. 24 (46.1\%) in REG-to-FTD/TPI. Haematologic toxicities $\mathrm{G} 3 / \mathrm{G} 4$, occurred more in pts treated with FTD/TPIto-REG $(30.7 \%$ ) (neutropenia $21.1 \%$, anemia $5.7 \%$ and leucopenia $1.9 \%$ ) than in those treated with the reverse sequence $(15.3 \%$ ) (neutropenia $9.6 \%$, anemia $3.8 \%$ and leucopenia $1.9 \%$ ), whereas non haematologic toxicities G3/G4 were more heavy in REG-to-FTD/TPI sequence $(30.7 \%$ ) (fatigue $15.3 \%$, hand-foot skin reaction $11.5 \%$ and liver dysfunction $3.8 \%$ ) than in the reverse sequence treatment $(21.1 \%$ ) (fatigue $9.6 \%$, hand-foot skin reaction $0 \%$ and liver dysfunction $0 \%$ ). However, only $2 \%$ of pts experienced febrile G4 neutropenia and received at least one dose of granulocyte colony-stimulating factor in the FTD/TDI-to-REG sequence exclusively. In $36.7 \%$ and $57.1 \%$ of pts treated with FTD/TPI and REG respectively, the doses were reduced for toxicity as follows: $-15 \%$ in $33.3 \%,-20 \%$ in $38.8 \%,-25 \%$ in $27.6 \%$ of pts in therapy with FTD/TPI, $-50 \%$ in $60.7 \%$ and $-25 \%$ in $39.2 \%$ of pts who received REG. There was no discontinuation of treatment due to toxicity. No therapy related death was reported. When analysed by age, $34.6 \%$ of pts aged $>70$ yrs $(44.4 \%$ in the REG-to-FTD/TPI group and $55.5 \%$ in the reverse group) and $65.3 \%$ of those aged $\leq 70$ yrs $(37.5 \%$ in the REG-to-FTD/TPI group and $62.5 \%$ in the reverse group) experienced at least one drug-related G3/G4 toxicity. Efficacy outcomes are shown in Table III. Median OS for the pts included in the FTP/TPI-to-REG group was 20 mos $(95 \% \mathrm{CI}=16.7-23.3) v s$. 27 mos of those treated with the reverse sequence (95\% CI=17.8-36.2). After $2 \mathrm{yrs}, 43.6 \%$ of pts treated with the FTP/TDI-to-REG sequence were still alive in comparison with $54.5 \%$ of patients treated with the reverse sequence $(p=0.81)$ (Figure 2). No differences were found in mPFS: 8 mos with FTD/TPI-to-REG $(95 \% \mathrm{CI}=6-10)$ vs. 9 mos with REG-to-FTD/TPI (95\%CI=7.2-10.8). Best 1-yr mPFS rate (25.7\%) was observed with the REG-to-FTP/TDI sequence $v s$. the reverse sequence $(12.4 \%)(p=0.32)$ (Figure 3). When analysed by baseline characteristic subgroups, mPFS was higher in pts aged $>70$ yrs than those $\leq 70$ yrs (11 versus 8 mos), in pts with primary tumour site of the rectum and then, in descending order, of the left and right colon (11 vs. 9 vs. 8 mos respectively) and moderately higher in pts with baseline ECOG PS 0 and 1 (both 9 mos) versus those with ECOG PS 2 (7 mos). Regarding the REG-FTD/TPI sequence, we observed a mPFS higher than that of the reverse sequence in pts with an ECOG PS 0 at baseline (11 vs. 7 mos), age $\leq 70$ (9 vs. 7.5 mos), wild type RAS status (10 vs. 8 mos), mutant type RAS status ( $9 v s .7$ mos), the site of the primary tumor on the left (10 vs. 7 mos), liver + other metastases (10 vs. 7 mos) and in pts with metastases other than the liver (10 vs. $8.5 \mathrm{mos})$. The reverse sequence showed a noteworthy mPFS concerning pts with an ECOG PS 1 at baseline (11 vs. 8 mos), age $>70$ yrs (11 vs. 9 mos) and only liver metastases (10.5 vs. 
Table I. Baseline patients' characteristics and treatments data.

\begin{tabular}{|c|c|c|c|c|c|}
\hline & \multicolumn{2}{|c|}{ FTD/TPI to REG } & \multicolumn{2}{|c|}{ REG to FTD/TPI } & \multirow[b]{2}{*}{$p$-Value } \\
\hline & $\mathrm{N}$ & $\%$ & $\mathrm{~N}$ & $\%$ & \\
\hline Total & 29 & 59.1 & 20 & 40.8 & \\
\hline Age, median (years) [range] & $68[48-83]$ & - & $64[45-80]$ & - & \\
\hline Gender & & & & & - \\
\hline Female & 6 & 20.6 & 11 & 55.0 & \\
\hline Male & 23 & 79.3 & 9 & 45.0 & \\
\hline$R A S$ status & & & & & 0.09 \\
\hline Wild type & 10 & 34.4 & 8 & 40.0 & \\
\hline Mutant type & 18 & 62.0 & 12 & 60.0 & \\
\hline Not tested & 1 & 3.4 & 0 & 0 & \\
\hline Tumor location & & & & & 0.92 \\
\hline Right side & 16 & 55.1 & 5 & 25.0 & \\
\hline Left side & 8 & 27.5 & 11 & 55.0 & \\
\hline Rectum & 5 & 17.2 & 4 & 20.0 & \\
\hline PS ECOG & & & & & 0.07 \\
\hline 0 & 12 & 41.3 & 8 & 40.0 & \\
\hline 1 & 14 & 48.2 & 11 & 55.0 & \\
\hline 2 & 3 & 10.3 & 1 & 5 & \\
\hline Metastatic disease sites & & & & & 0.98 \\
\hline Liver only & 4 & 13.7 & 5 & 25.0 & \\
\hline Liver + other & 8 & 27.5 & 9 & 45.0 & \\
\hline Other & 17 & 58.6 & 6 & 30.0 & \\
\hline Patients aged $>70$ years & 10 & 34.4 & 7 & 35.0 & - \\
\hline Patients aged $\leq 70$ years & 19 & 65.5 & 13 & 65.0 & \\
\hline CT $1^{\circ}$ line regimen & & & & & 0.07 \\
\hline Fluoropyrimidine alone & 2 & 6.8 & 1 & 5.0 & \\
\hline Doublet chemotherapy & 25 & 86.2 & 16 & 80.0 & \\
\hline Triplet chemotherapy & 2 & 6.8 & 3 & 15.0 & \\
\hline CT $2^{\circ}$ line regimen & & & & & 0.03 \\
\hline Fluoropyrimidine alone & 3 & 10.3 & 2 & 10.0 & \\
\hline Doublet chemotherapy & 26 & 89.6 & 17 & 85.0 & \\
\hline Triplet chemotherapy & 0 & 0 & 1 & 5.0 & \\
\hline СТ $3^{\circ}$ line regimen & & & & & 0.10 \\
\hline Fluoropyrimidine alone & 5 & 17.2 & 4 & 20.0 & \\
\hline Doublet chemotherapy & 3 & 10.3 & 3 & 15.0 & \\
\hline Triplet chemotherapy & 0 & 0 & 0 & 0 & \\
\hline Biological agents $1^{\circ}$ line & & & & & 0.03 \\
\hline Cetuximab use & 5 & 17.2 & 3 & 15.0 & \\
\hline Panitumumab use & 4 & 13.7 & 1 & 5.0 & \\
\hline Bevacizumab use & 16 & 55.1 & 15 & 75.0 & \\
\hline Biological agents $2^{\circ}$ line & & & & & 0.01 \\
\hline Cetuximab use & 1 & 3.4 & 2 & 10.0 & \\
\hline Panitumumab use & 0 & 0 & 0 & 0 & \\
\hline Bevacizumab use & 11 & 37.9 & 8 & 40.0 & \\
\hline Aflibercept use & 9 & 31.0 & 6 & 30.0 & \\
\hline Biological agents $3^{\circ}$ line & & & & & 0.33 \\
\hline Cetuximab use & 1 & 3.4 & 2 & 10.0 & \\
\hline Panitumumab use & 1 & 3.4 & 1 & 5.0 & \\
\hline Bevacizumab use & 0 & 0 & 0 & 0 & \\
\hline
\end{tabular}

8 mos). Median PFS in both sequences did not appear to be impacted by right primary tumour site $(8 \mathrm{vs} .8 .5 \mathrm{mos})$ and rectum primary tumour site (11 vs. $11 \mathrm{mos})$. Finally, ORR was $6.9 \%$ for the FTD/TPI-to-REG sequence $v s .5 .0 \%$ with the reverse sequence $(p=0.72)$. No complete responses were achieved. The DCR was estimated to be $45.0 \%$ for pts treated with the REG-to-FTD/TPI sequence versus $24.1 \%$ in those treated with the reverse sequence $(p=0.18)$. 
Table II. Drug-related toxicities.

\begin{tabular}{|c|c|c|c|c|c|}
\hline & \multicolumn{2}{|c|}{ FTD/TPI to REG } & \multicolumn{2}{|c|}{ REG to FTD/TPI } & \multirow[b]{2}{*}{ p-Value } \\
\hline & $\mathrm{N}$ & $\%$ & $\mathrm{~N}$ & $\%$ & \\
\hline All events G3/G4 (N=52) & 28 & 53.8 & 24 & 46.1 & - \\
\hline Haematologic toxicities & 16 & 30.7 & 8 & 15.3 & 0.02 \\
\hline Anemia & 3 & 5.7 & 2 & 3.8 & \\
\hline Leucopenia & 1 & 1.9 & 1 & 1.9 & \\
\hline Neutropenia & 11 & 21.1 & 5 & 9.6 & \\
\hline Thrombocytopenia & 1 & 1.9 & 0 & 0 & \\
\hline Non haematologic toxicities & 11 & 21.1 & 17 & 30.7 & 0.49 \\
\hline Fatigue & 5 & 9.6 & 8 & 15.3 & \\
\hline Hand-Foot skin reaction & 0 & 0 & 6 & 11.5 & \\
\hline Liver dysfunctions & 0 & 0 & 2 & 3.8 & \\
\hline Skin disorders & 2 & 3.8 & 0 & 0 & \\
\hline Diarrhoea & 2 & 3.8 & 1 & 1.9 & \\
\hline Others & 2 & 3.8 & 0 & 0 & \\
\hline
\end{tabular}

FTD/TPI: Trifluridine/tipiracil; REG: regorafenib.

Table III. Efficacy outcomes.

\begin{tabular}{|c|c|c|c|c|c|c|c|c|c|c|c|c|}
\hline & \multicolumn{4}{|c|}{ OS } & \multicolumn{4}{|c|}{ PFS } & \multicolumn{2}{|c|}{ ORR } & \multicolumn{2}{|c|}{ DCR } \\
\hline & $\begin{array}{l}\mathrm{mOS} \\
(\mathrm{mos})\end{array}$ & $95 \% \mathrm{CI}$ & $\begin{array}{c}2-y r s \\
(\%)\end{array}$ & $p$-Value & $\begin{array}{l}\mathrm{mPFS} \\
(\mathrm{mos})\end{array}$ & $95 \% \mathrm{CI}$ & $\begin{array}{l}1-\mathrm{yr} \\
(\%)\end{array}$ & $p$-Value & $\begin{array}{c}\mathrm{PR}+\mathrm{CR} \\
(\%)\end{array}$ & $p$-Value & $\begin{array}{c}\mathrm{PR}+\mathrm{CR}+ \\
\mathrm{SD}(\%)\end{array}$ & $p$-Value \\
\hline FTP/TPI-to-REG & 20 & $16.7-23.3$ & 43.6 & 0.81 & 8 & $6-10$ & 12.4 & 0.32 & 6.9 & 0.72 & 24.1 & 0.18 \\
\hline REG-to-FTP/TPI & 27 & $17.8-36.2$ & 54.5 & & 9 & $7.2-10.8$ & 25.7 & & 5.0 & & 45.0 & \\
\hline
\end{tabular}

FTD/TPI: Trifluridine/tipiracil; REG: regorafenib; CI: confidence interval; OS: overall survival; PFS: progression-free survival; ORR: overall response rate; DCR: disease control rate; PR: partial response; CR: complete response; SD: stable disease.

\section{Discussion}

Our retrospective analysis suggests that regorafenib and FTD/TPI regardless of the order of their administration, can be recommended for patients with refractory mCRC in the real-life setting. The efficacy and safety experiences in pts treated in late-lines, are very important for clinicians precisely because, unlike the phase III studies, the particularities of pts such as the ECOG PS, may be different in everyday clinical practice. Likewise, the administration of drugs should be established on a careful evaluation of the patients' conditions with the aim of prolonging their survival and not worsening the PS rather than seeking an objective response of the disease. As is widely known, both agents have been reported to show similar efficacy but different toxicity profiles in retrospective studies $(16,19,20)$ but to date, it is unconfirmed which should be used first and which outcome could be achieved if administered sequentially. The median PFS and OS of REG monotherapy reported in the phase III CORRECT study were 1.9 months and 6.4 months (10) while in the phase
III RECOURSE study the median PFS and OS of FTD/TPI monotherapy were 2.0 and 7.1 mos respectively (11). In our study, the OS seemed to be higher in the REG-to-FTP/TDI group than in the reverse group. The sequence of the two drug administrations that were compared regardless of order, although not statistically significant, revealed a 7-month survival advantage in the REG-to-FTD/TPI group. However, there was no difference in terms of PFS. The two sequences were almost equivalent in terms of objective responses with no complete response. On the other hand, there was an interesting advantage of $20.9 \%$ in terms of disease control rate in favor of the REG-to-FTD/TPI sequence that should not to be underestimated, as these pts present with a metastatic and refractory disease to multiple treatments, have a poor prognosis, and a not always optimal performance status. Our results showed that subsequent additional administration of FTP/TDI after REG may be more effective for prolonging OS and for disease controlling in mCRC refractory to previous treatments. The subgroup analysis revealed a higher mPFS if REG was administered first in the sequence in pts aged $\leq 70$ 


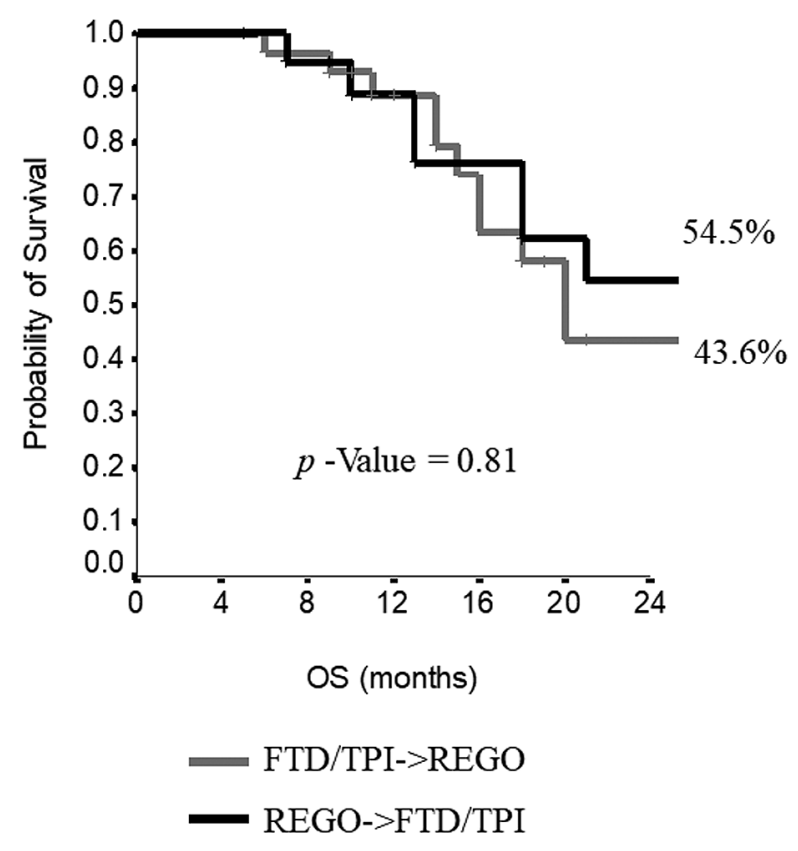

Figure 2. Overall survival (OS) curve comparing trifluridine/tipiracil (FTD/TPI)-to-regorafenib (REG) sequence to the reverse REG-toFTD/TPI sequence. Grey line: FTD/TPI-to-REG sequence, black line: REG-to-FTD/TPI sequence; after 2 years, $43.6 \%$ of patients treated with the FTP/TDI-to-REG sequence were still alive in comparison with $54.5 \%$ of patients treated with the reverse sequence $(p=0.81)$.

yrs, ECOG PS 0, left primary tumour site and with liver + other metastases. A longer mPFS was observed if FTD/TPI was admnistered first in pts with ECOG PS 1 and with only liver metastases. However, these are non-statistically significant data that were collected from a limited sample of 49 patients but it could already provide a clear indication and suggestion on which drug is better to be administered first when the clinician needs to treat a patient with a refractory mCRC but with a good PS and the disease is still therapeutically attackable in a systemic way. After all, the safety profiles of FTD/TPI and REG administered sequentially were concordant with those reported in previous studies $(10$, 11). The main toxic events observed in the FTD/TPI-to-REG sequence were confirmed to be haematological, mainly neutropenia and anemia with a febrile neutropenia estimated at around $2 \%$ and therefore is considered tolerable. The main notable adverse events in the REG group were hand-foot syndrome and fatigue. Some patients started REG as a salvage therapy with a reduced dose, and the group that started with the reduced dose of $120 \mathrm{mg}$ had the best mPFS of 12.5 months. Although starting REG therapy with reduced doses is not recommended by everyone, our study confirms that optimizing the REG dose can be considered reasonable even as evidenced by the ReDOS study $(18,21)$. In this study, the

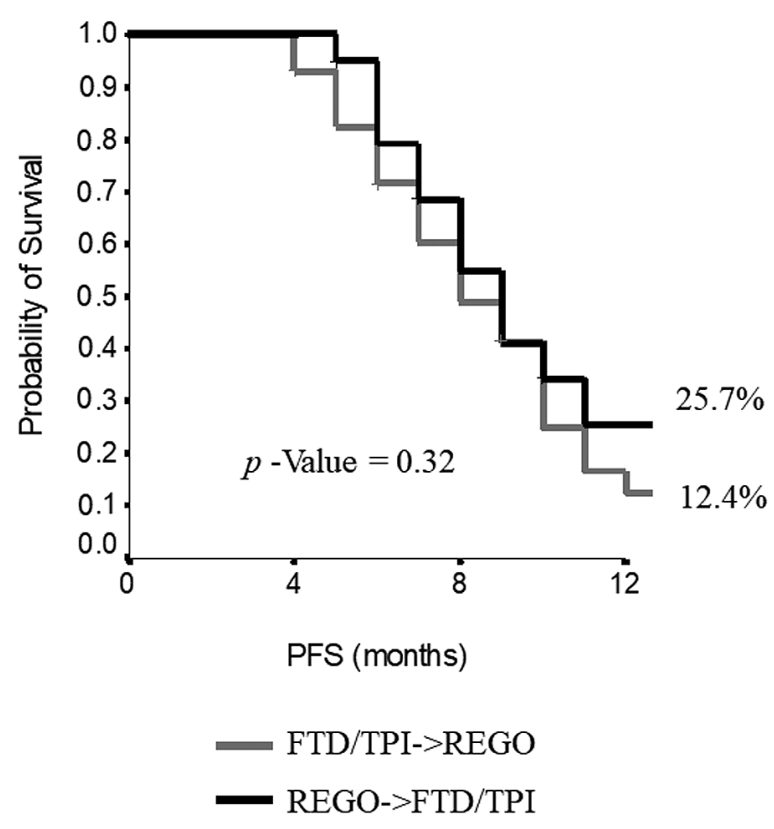

Figure 3. Progression-free survival (PFS) curve comparing trifluridine/tipiracil (FTD/TPI)-to-regorafenib (REG) sequence to the reverse REG-to-FTD/TPI sequence. Grey line: FTD/TPI-to-REG sequence, black line: REG-to-FTD/TPI sequence; best 1-year mPFS rate $(25.7 \%)$ was observed with the REG-to-FTP/TDI sequence vs. the reverse sequence FTD/TPI-to-REG (12.4\%) ( $p=0.32)$.

safety profiles of REG and FTP/TDI were broadly consistent with those of previous pivotal studies $(10,11,22)$. In addition, the incidence of hand-foot syndrome and fatigue in patients given regorafenib was not significantly increased even though the drug was used after FTD/TPI, while, conversely, the rates of myelosuppression, including anemia and neutropenia in the patients who received FTD/TPI were not major in patients with previous regorafenib treatment. No discontinuation of treatment due to toxic effects was observed. The incidence of toxic effects was not increased in patients initiating the therapy sequence with FTP/TDI provided REG was administered with the escalating-dose. These results indicate that both drugs can be safely administered before or after indifferently without additional toxicity. In short, we conclude that the prevalent toxicities of the entire therapeutic sequence appear related to the first drug administered. Our analysis has some limitations, such as the inclusion of only a relatively small number of patients collected from a limited number of institutions of Lazio region. However, in its small way, our study has retrospectively observed what happens in daily clinical practice and began to answer the questions that the oncologist asks before deciding which drug to administer alone or in sequence in third or later lines in pts with mCRC refractory to standard chemotherapy. 


\section{Conclusion}

Our study demonstrated the efficacy and manageable toxicities of the therapeutic sequences FTD/TPI to REG and REG to FTD/TPI in the real world context. REG and FTD/TPI have similar survival effects but the sequential use of both drugs is useful for prolonging OS without acting on tumor shrinkage. Future investigations about their optimal sequential order of administration and the efficacy and safety of both drugs are needed. With the sample size limit, we conclude that in our real-world experience, REGto-FTD/TPI sequence and the reverse sequence could extend survival while only REG-to-FTD/TPI stabilizes cancer growth. The prevalent toxicities appear to be related to the first drug administered in the sequence. It may be important to decide which drug in the sequence should be given in the beginning. Further clinical studies are needed to help us better understand the use of these lines of therapy in the real world practice and examine more current treatment options.

\section{Conflicts of Interest}

The Authors declare that they have no competing interests in relation to this study.

\section{Authors' Contributions}

Conceptualization: CS; Data Curation: CS; Formal Analysis: CS, IS; Investigation: CS, DG, FZ, MGM, RG, RS, GS, SDM, AC; Resources: CS; Validation: CS; Visualization: CS; Writing Original Draft Preparation: CS; Writing - Review \& Editing: CS, EMR; All Authors have read and approved the final manuscript.

\section{References}

1 Sung H, Ferlay J, Siegel RL, Laversanne M, Soerjomataram I, Jemal A and Bray F: Global cancer statistics 2020: GLOBOCAN estimates of incidence and mortality worldwide for 36 cancers in 185 countries. CA Cancer J Clin : 2021. PMID: 33538338. DOI: $10.3322 /$ caac. 21660

2 Le D, Kavan P, Kim T, Burge M, Van cutsem E, Hara H, Boland P, Van laethem J, Geva R, Taniguchi H, Crocenzi T, Sharma M, Atreya C, Diaz L, Liang L, Marinello P, Dai T and O'neil B: KEYNOTE-164: Pembrolizumab for patients with advanced microsatellite instability high (MSI-H) colorectal cancer. Journal of Clinical Oncology 36(15_suppl): 3514-3514, 2019. DOI: 10.1200/JCO.2018.36.15_suppl.3514

3 André T, Shiu KK, Kim TW, Jensen BV, Jensen LH, Punt C, Smith D, Garcia-Carbonero R, Benavides M, Gibbs P, de la Fouchardiere C, Rivera F, Elez E, Bendell J, Le DT, Yoshino T, Van Cutsem E, Yang P, Farooqui MZH, Marinello P, Diaz LA Jr and KEYNOTE177 Investigators: Pembrolizumab in microsatellite-instability-high advanced colorectal cancer. N Engl J Med 383(23): 2207-2218, 2020. PMID: 33264544. DOI: 10.1056/NEJMoa2017699
4 Heinemann V, von Weikersthal LF, Decker T, Kiani A, VehlingKaiser U, Al-Batran SE, Heintges T, Lerchenmüller C, Kahl C, Seipelt G, Kullmann F, Stauch M, Scheithauer W, Hielscher J, Scholz M, Müller S, Link H, Niederle N, Rost A, Höffkes HG, Moehler M, Lindig RU, Modest DP, Rossius L, Kirchner T, Jung A and Stintzing S: Folfiri plus cetuximab versus folfiri plus bevacizumab as first-line treatment for patients with metastatic colorectal cancer (FIRE-3): A randomised, open-label, phase 3 trial. Lancet Oncol 15(10): 1065-1075, 2014. PMID: 25088940. DOI: $10.1016 / \mathrm{S} 1470-2045(14) 70330-4$

5 Venook AP, Niedzwiecki D, Lenz HJ, Innocenti F, Fruth B, Meyerhardt JA, Schrag D, Greene C, O'Neil BH, Atkins JN, Berry S, Polite BN, O’Reilly EM, Goldberg RM, Hochster HS, Schilsky RL, Bertagnolli MM, El-Khoueiry AB, Watson P, Benson AB 3rd, Mulkerin DL, Mayer RJ and Blanke C: Effect of first-line chemotherapy combined with cetuximab or bevacizumab on overall survival in patients with KRAS wildtype advanced or metastatic colorectal cancer: A randomized clinical trial. JAMA 317(23): 2392-2401, 2017. PMID: 28632865. DOI: $10.1001 /$ jama.2017.7105

6 Loupakis F, Cremolini C, Masi G, Lonardi S, Zagonel V, Salvatore L, Cortesi E, Tomasello G, Ronzoni M, Spadi R, Zaniboni A, Tonini G, Buonadonna A, Amoroso D, Chiara S, Carlomagno C, Boni C, Allegrini G, Boni L and Falcone A: Initial therapy with folfoxiri and bevacizumab for metastatic colorectal cancer. N Engl J Med 371(17): 1609-1618, 2014. PMID: 25337750. DOI: 10.1056/NEJMoa1403108

7 Tabernero J, Yoshino T, Cohn AL, Obermannova R, Bodoky G, Garcia-Carbonero R, Ciuleanu TE, Portnoy DC, Van Cutsem E, Grothey A, Prausová J, Garcia-Alfonso P, Yamazaki K, Clingan PR, Lonardi S, Kim TW, Simms L, Chang SC, Nasroulah F and RAISE Study Investigators: Ramucirumab versus placebo in combination with second-line folfiri in patients with metastatic colorectal carcinoma that progressed during or after first-line therapy with bevacizumab, oxaliplatin, and a fluoropyrimidine (RAISE): A randomised, double-blind, multicentre, phase 3 study. Lancet Oncol 16(5): 499-508, 2015. PMID: 25877855. DOI: $10.1016 / \mathrm{S} 1470-2045(15) 70127-0$

8 Van Cutsem E, Tabernero J, Lakomy R, Prenen H, Prausová J, Macarulla T, Ruff P, van Hazel GA, Moiseyenko V, Ferry D, McKendrick J, Polikoff J, Tellier A, Castan R and Allegra C: Addition of aflibercept to fluorouracil, leucovorin, and irinotecan improves survival in a phase III randomized trial in patients with metastatic colorectal cancer previously treated with an oxaliplatin-based regimen. J Clin Oncol 30(28): 3499-3506, 2012. PMID: 22949147. DOI: 10.1200/JCO.2012.42.8201

9 Yamazaki K, Nagase M, Tamagawa H, Ueda S, Tamura T, Murata K, Eguchi Nakajima T, Baba E, Tsuda M, Moriwaki T, Esaki T, Tsuji Y, Muro K, Taira K, Denda T, Funai S, Shinozaki K, Yamashita H, Sugimoto N, Okuno T, Nishina T, Umeki M, Kurimoto T, Takayama T, Tsuji A, Yoshida M, Hosokawa A, Shibata Y, Suyama K, Okabe M, Suzuki K, Seki N, Kawakami K, Sato M, Fujikawa K, Hirashima T, Shimura T, Taku K, Otsuji T, Tamura F, Shinozaki E, Nakashima K, Hara H, Tsushima T, Ando M, Morita S, Boku N and Hyodo I: Randomized phase III study of bevacizumab plus folfiri and bevacizumab plus mfolfox 6 as first-line treatment for patients with metastatic colorectal cancer (WJOG4407G). Ann Oncol 27(8): 1539-1546, 2016. PMID: 27177863. DOI: 10.1093/annonc/mdw206 
10 Grothey A, Van Cutsem E, Sobrero A, Siena S, Falcone A, Ychou M, Humblet Y, Bouché O, Mineur L, Barone C, Adenis A, Tabernero J, Yoshino T, Lenz HJ, Goldberg RM, Sargent DJ, Cihon F, Cupit L, Wagner A, Laurent D and CORRECT Study Group: Regorafenib monotherapy for previously treated metastatic colorectal cancer (CORRECT): An international, multicentre, randomised, placebo-controlled, phase 3 trial. Lancet 381(9863): 303-312, 2013. PMID: 23177514. DOI: 10.1016/S0140-6736(12)61900-X

11 Mayer RJ, Van Cutsem E, Falcone A, Yoshino T, GarciaCarbonero R, Mizunuma N, Yamazaki K, Shimada Y, Tabernero J, Komatsu Y, Sobrero A, Boucher E, Peeters M, Tran B, Lenz HJ, Zaniboni A, Hochster H, Cleary JM, Prenen H, Benedetti F, Mizuguchi H, Makris L, Ito M, Ohtsu A and RECOURSE Study Group: Randomized trial of TAS-102 for refractory metastatic colorectal cancer. N Engl J Med 372(20): 1909-1919, 2015. PMID: 25970050. DOI: 10.1056/NEJMoa1414325

12 Van Cutsem E, Cervantes A, Adam R, Sobrero A, Van Krieken JH, Aderka D, Aranda Aguilar E, Bardelli A, Benson A, Bodoky G, Ciardiello F, D'Hoore A, Diaz-Rubio E, Douillard JY, Ducreux M, Falcone A, Grothey A, Gruenberger T, Haustermans K, Heinemann V, Hoff P, Köhne CH, Labianca R, Laurent-Puig P, Ma B, Maughan T, Muro K, Normanno N, Österlund P, Oyen WJ, Papamichael D, Pentheroudakis G, Pfeiffer P, Price TJ, Punt C, Ricke J, Roth A, Salazar R, Scheithauer W, Schmoll HJ, Tabernero J, Taïeb J, Tejpar S, Wasan H, Yoshino T, Zaanan A and Arnold D: ESMO consensus guidelines for the management of patients with metastatic colorectal cancer. Ann Oncol 27(8): 13861422, 2016. PMID: 27380959. DOI: 10.1093/annonc/mdw235

13 NCCN Clinical Practice Guidelines in Oncology (NCCN Guidelines). Colon Cancer. Version 2.2021 - January 21, 2021. Available at: www.ncen.org [Last accessed on March 26, 2021]

14 Li J, Qin S, Xu R, Yau TC, Ma B, Pan H, Xu J, Bai Y, Chi Y, Wang L, Yeh KH, Bi F, Cheng Y, Le AT, Lin JK, Liu T, Ma D, Kappeler C, Kalmus J, Kim TW and CONCUR Investigators: Regorafenib plus best supportive care versus placebo plus best supportive care in Asian patients with previously treated metastatic colorectal cancer (CONCUR): A randomised, doubleblind, placebo-controlled, phase 3 trial. Lancet Oncol 16(6): 619-629, 2015. PMID: 25981818. DOI: 10.1016/S14702045(15)70156-7

15 Xu J, Kim TW, Shen L, Sriuranpong V, Pan H, Xu R, Guo W, Han SW, Liu T, Park YS, Shi C, Bai Y, Bi F, Ahn JB, Qin S, Li Q, Wu C, Ma D, Lin D and Li J: Results of a randomized, double-blind, placebo-controlled, phase III trial of trifluridine/tipiracil (TAS-102) monotherapy in Asian patients with previously treated metastatic colorectal cancer: The TERRA study. J Clin Oncol 36(4): 350-358, 2018. PMID: 29215955. DOI: $10.1200 / \mathrm{JCO} .2017 .74 .3245$

16 Moriwaki T, Fukuoka S, Taniguchi H, Takashima A, Kumekawa Y, Kajiwara T, Yamazaki K, Esaki T, Makiyama C, Denda T, Satake H, Suto T, Sugimoto N, Enomoto M, Ishikawa T, Kashiwada T, Sugiyama M, Komatsu Y, Okuyama H, Baba E, Sakai D, Watanabe T, Tamura T, Yamashita K, Gosho M and Shimada Y: Propensity score analysis of regorafenib versus trifluridine/tipiracil in patients with metastatic colorectal cancer refractory to standard chemotherapy (REGOTAS): A Japanese society for cancer of the colon and rectum multicenter observational study. Oncologist 23(1): 7-15, 2018. PMID: 28894015. DOI: $10.1634 /$ theoncologist.2017-0275
17 Patel AK, Barghout V, Yenikomshian MA, Germain G, Jacques P, Laliberté F and Duh MS: Real-world adherence in patients with metastatic colorectal cancer treated with trifluridine plus tipiracil or regorafenib. Oncologist 25(1): e75-e84, 2020. PMID: 31591140. DOI: 10.1634/theoncologist.2019-0240

18 Bekaii-Saab TS, Ou FS, Ahn DH, Boland PM, Ciombor KK, Heying EN, Dockter TJ, Jacobs NL, Pasche BC, Cleary JM, Meyers JP, Desnoyers RJ, McCune JS, Pedersen K, Barzi A, Chiorean EG, Sloan J, Lacouture ME, Lenz HJ and Grothey A: Regorafenib dose-optimisation in patients with refractory metastatic colorectal cancer (ReDOS): A randomised, multicentre, open-label, phase 2 study. Lancet Oncol 20(8): 1070-1082, 2019. PMID: 31262657. DOI: 10.1016/S14702045(19)30272-4

19 Sueda T, Sakai D, Kudo T, Sugiura T, Takahashi H, Haraguchi N, Nishimura J, Hata T, Hayashi T, Mizushima T, Doki Y, Mori $\mathrm{M}$ and Satoh T: Efficacy and safety of regorafenib or TAS-102 in patients with metastatic colorectal cancer refractory to standard therapies. Anticancer Res 36(8): 4299-4306, 2016. PMID: 27466548.

20 Masuishi T, Taniguchi H, Hamauchi S, Komori A, Kito Y, Narita Y, Tsushima T, Ishihara M, Todaka A, Tanaka T, Yokota T, Kadowaki S, Machida N, Ura T, Fukutomi A, Ando M, Onozawa Y, Tajika M, Yasui H, Muro K, Mori K and Yamazaki K: Regorafenib versus trifluridine/tipiracil for refractory metastatic colorectal cancer: A retrospective comparison. Clin Colorectal Cancer 16(2): e15-e22, 2017. PMID: 27670892. DOI: 10.1016/ j.clcc. 2016.07 .019

21 Osawa $\mathrm{H}$ : Response to regorafenib at an initial dose of $120 \mathrm{mg}$ as salvage therapy for metastatic colorectal cancer. Mol Clin Oncol 6(3): 365-372, 2017. PMID: 28451414. DOI: 10.3892/mco.2017. 1145

22 Grothey A, Falcone A, Humblet Y, Bouche O, Mineur L, Adenis A, Tabernero J, Yoshino T, Lenz H, Goldberg R, Huang L, Wagner A and Van cutsem E: Characteristics of patients (pts) with metastatic colorectal cancer (mCRC) treated with regorafenib (REG) who had progression-free survival $(\mathrm{PFS})>4$ months $(\mathrm{m})$ : Subgroup analysis of the phase 3 correct trial. Annals of Oncology 27: vi170, 2020. DOI: 10.1093/annonc/mdw370.64

23 Bekaii-Saab T, Kim R, Kim TW, O’Connor JM, Strickler JH, Malka D, Sartore-Bianchi A, Bi F, Yamaguchi K, Yoshino T and Prager GW: Third- or later-line therapy for metastatic colorectal cancer: Reviewing best practice. Clin Colorectal Cancer 18(1): e117-e129, 2019. PMID: 30598357. DOI: 10.1016/j.clcc.2018.11.002

24 Tanaka A, Sadahiro S, Suzuki T, Okada K, Saito G and Miyakita $\mathrm{H}$ : Retrospective study of regorafenib and trifluridine/tipiracil efficacy as a third-line or later chemotherapy regimen for refractory metastatic colorectal cancer. Oncol Lett 16(5): 65896597, 2018. PMID: 30344762. DOI: 10.3892/ol.2018.9421

25 Patel AK, Barghout V, Yenikomshian MA, Germain G, Jacques $\mathrm{P}$, Laliberté $\mathrm{F}$ and Duh MS: Real-world adherence in patients with metastatic colorectal cancer treated with trifluridine plus tipiracil or regorafenib. Oncologist 25(1): e75-e84, 2020. PMID: 31591140. DOI: 10.1634/theoncologist.2019-0240

26 Ogata M, Kotaka M, Ogata T, Hatachi Y, Yasui H, Kato T, Tsuji A and Satake H: Regorafenib vs trifluridine/tipiracil for metastatic colorectal cancer refractory to standard chemotherapies: A multicenter retrospective comparison study in Japan. PLoS One 15(6): e0234314, 2020. PMID: 32530932. DOI: 10.1371 /journal.pone. 0234314 
27 Moriwaki T, Fukuoka S, Taniguchi H, Takashima A, Kumekawa Y, Kajiwara T, Yamazaki K, Esaki T, Makiyama C, Denda T, Satake H, Suto T, Sugimoto N, Enomoto M, Ishikawa T, Kashiwada T, Sugiyama M, Komatsu Y, Okuyama H, Baba E, Sakai D, Watanabe T, Tamura T, Yamashita K, Gosho M and Shimada Y: Propensity score analysis of regorafenib versus trifluridine/tipiracil in patients with metastatic colorectal cancer refractory to standard chemotherapy (REGOTAS): A Japanese society for cancer of the colon and rectum multicenter observational study. Oncologist 23(1): 7-15, 2018. PMID: 28894015. DOI: 10.1634/theoncologist.2017-0275

28 Bachet JB, Wyrwicz L, Price T, Cremolini C, Phelip JM, Portales F, Ozet A, Cicin I, Atlan D, Becquart M, Vidot L, Mounedji N, Van Cutsem E, Taieb J and Falcone A: Safety, efficacy and patient-reported outcomes with trifluridine/tipiracil in pretreated metastatic colorectal cancer: Results of the PRECONNECT study. ESMO Open 5(3): e000698, 2020. PMID: 32487542. DOI: 10.1136/esmoopen-2020-000698

29 Papadimitriou M and Papadimitriou CA: Antiangiogenic tyrosine kinase inhibitors in metastatic colorectal cancer: Focusing on regorafenib. Anticancer Res 41(2): 567-582, 2021. PMID: 33517262. DOI: 10.21873 /anticanres.14809

Received March 20, 2021

Revised March 27, 2021

Accepted March 29, 2021 